Review Article

\title{
PHARMACEUTICAL WORLD WITH INNOVATIVE APPROACHES-A REVIEW
}

\author{
HARSH RASTOGI ${ }^{1}$, HRISHAV DAS PURKAYASTHA ${ }^{2}$, SHALINI TIWARI ${ }^{3}$
}

${ }^{1}$ Ram-Eesh Institute of Vocational and Technical Education (Department of Pharmacy), Greater Noida, U. P., ${ }^{2}$ Acharya and BM Reddy College of Pharmacy, Hesarghatta Main Road, Bengaluru, Karnataka, ${ }^{3}$ Sunderdeep Pharmacy College, Dasna-Delhi Highway, Ghaziabad, U. P. Email: reviewarticles100@gmail.com

Received: 14 May 2019, Revised and Accepted: 15 Jul 2019

\begin{abstract}
An existing drug in the conventional dosage form is evolution in to novel drug delivery system which can significantly enhances and improves the performance of drug in terms of drug safety, drug efficacy as well as patient compliance. With the help of appropriate novel approaches, an existing drug molecule can get a new life and can be a major advance solution for the problems towards the release of drug at specific site and specific rate. The main aim of novel drug delivery system is to minimize the drug loss and drug degradation which can be achieved by targeting the drug to the site of interest. With the help of soluble or insoluble biodegradable natural and synthetic polymers different novel formulations can be formulated as microparticles, microcapsules, liposomes, neosomes, micelles etc. Targeting is based on active targeting and passive targeting mechanisms for drug release. This review covers the basic information on Novel Drug Delivery Systems (NDDS).
\end{abstract}

Keywords: Conventional drug delivery system, Novel drug delivery system, Micelles, Neosomes, Microparticles

(C) 2019 The Authors. Published by Innovare Academic Sciences Pvt Ltd. This is an open-access article under the CC BY license (http://creativecommons.org/licenses/by/4.0/] DOI: http://dx.doi.org/10.22159/ijcpr.2019v11i5.35700

\section{INTRODUCTION [1-6]}

Novel Drug delivery System (NDDS) refers to the system which helps in transporting a pharmaceutical compound in body which can safely achieve its desired therapeutic effects. It involves site-targeting within the body, or facilitating systemic pharmacokinetics; in any case, it is typically concerned with both quantity and duration of presence of drug and it is advanced drug delivery system which improves drug potency, drug release to provide a sustained therapeutic effect, provide safety, finally drug can be targeted specifically to a specific site. Among drug carrier one can name soluble polymers, microparticles made of insoluble (or) biodegradable natural and synthetic polymers, microcapsules, cells, cell ghosts, lipoproteins, liposomes and micelles. Previously the scientists use to alter the therapeutics of drug by altering the drug pharmacodynamics but in novel drug delivery system the therapeutics of drug based on altering the pharmacokinetics of the drug by using different methods. Basically the approach to deliver the drug in the body is based on two basic mechanisms:

\section{Active targeting}

Inactive targeting, the pharmacokinetics of the drug gets altered and modified carriers are employed to make it more specific to the target and active response can be observed at target site.

\section{Passive targeting}

In passive targeting, the natural course of bio-distribution of the carrier is employed. In passive targeting colloidal carriers are used.

The novel and innovative approaches are explained as follows:

\section{Drug delivery carriers [7]}

Colloidal drug carrier systems as micellar solutions, liquid crystal dispersions, vesicles, nanoparticle dispersions. Colloidal drug delivery system consists of small particles of 10-400 nm diameter. When developing these carriers, the aim is to obtain optimized drug loading and release properties, long shelf-life and low or no toxicity. The incorporated drug participates in the microstructure of the system, and may even influence it due to molecular interactions, or if the drug possesses amphiphilic and/or mesogenic properties.

\section{Hydrogels [8]}

Hydrogels are three-dimensional, hydrophilic, polymeric network capable of absorbing large amounts of biological fluids. The mechanism of hydrogels is depend on reservoir-based drug release, controlled release or as swelling-controlled release devices. These networks are composed of homopolymers or copolymers and are insoluble due to the presence of chemical cross-links (tie-points, junctions), or physical cross-links, such as entanglements or crystallites. Hydrogels exhibit a thermodynamic compatibility with water, which allows them to swell in aqueous media. On the forefront of controlled drug delivery, in these systems, release can be designed to occur within specific areas of the body (e. g., within a certain $\mathrm{pH}$ of the digestive tract) or also via specific sites (adhesive or cell-receptor specific gels via tethered chains from the hydrogel surface).

\section{Liposomes [8-10]}

Liposomes are consist of one or more phospholipids bilayer. The polar character of the liposomal core enables polar drug molecules to be encapsulated. Liposomes can also reduce or eliminate certain common side effects of cancer treatment such as nausea and hair loss. Amphiphilic and lipophilic molecules are solubilized within the phospholipids bilayer according to their affinity towards the phospholipids. Channel proteins can be incorporated without loss of their activity within the hydrophobic domain of vesicle membranes, acting as a size-selective filter, only allowing passive diffusion of small solutes such as ions, nutrients and antibiotics.

\section{Dendrimers [9]}

Dendrimers are nanometer-sized, highly branched monodisperse and synthetic nanoparticles that are approximately 5-10 nm in diameter. They consist of a polymeric layer surrounded by a central core. The core, together with the internal units, determine the nanocavities and their solubilizing properties and the external groups determine the solubility and chemical behavior of polymers. It contains many different sites to which drugs may be attached and also attachment sites for materials such as PEG which can be used to modified the way of dendrimer which interacts with body. PEG can be attached to dendrimer to prevent the body's defense mechanism for detecting it, thereby slowing the process of break down which help in cancer treatment and its many branches allow other molecules to easily attach to its surface.

\section{Nanoparticles [2, 10-13]}

Nanoparticles as nanospheres and nanocapsules of size 10-200 nm are in the solid-state and can be amorphous as well in crystalline form. Nanoparticles can absorb or encapsulate a drug or a mixture of 
drugs and helps in protecting it against from chemical and enzymatic degradation. In recent years, biodegradable polymeric nanoparticles can be easily applied in the controlled release of drugs, in targeting particular organ/or tissue, as carriers of DNA in gene therapy, and in their abilities to deliver proteins peptides and genes through peroral route.

\section{Classification of nano-formulations (nanoparticles)}

A) Nanotubes-Hallow cylinder consists of carbon atoms called nanotubes. They can also be filled and sealed, forming test tubes or potential drug delivery devices.

B) Nanowires-Glowing silica nanowire is wrapped around a single stand of human hair comprises nanowire. It looks delicate. It is about five times smaller than virus applications for nanowires include the early sensing of breast and ovarian malignancies.

C) Nanocantilever-The honeycomb mesh behind this tiny carbon cantilever is surface of fly's eye. Cantilevers are beams anchored at only one end. In nanoworld they function as sensors ideal for detecting the presence of extremely small molecules in biological fluid.

D) Nanoshells-Hallow silica spheres covered with gold layer called nanoshells. Scientists can attach antibodies to their surfaces enabling the shells to target certain shells such as cancer cells.

E) Quantum dots-Quantum dots are minuscule semiconductor particles that can serve as sign pots of certain type of cells or molecules in the body. They can do this because they emit different wavelengths of radiations depending upon the type of cadmium used in their cores. Cadmium sulfide for ultraviolet to blue, cadmium selinide for most of the visible spectrum and cadmium telluride for far infrared and near-infrared.

F) Nano pores-Nano pores have cancer research and treatment applications. Engineered into particles, they are holes that are so tiny that DNA molecules can pass through them one strand at a time allowing for highly precise and efficient DNA sequencing. By engineering nanopores into surface of drug capsule that are only slightly larger than medicines molecular structure, drug manufacturers can also use nanopores to control the rate of drug's diffusion in body.

G) Gold nanoparticles-These nanoparticles seen in transmission electron micrograph image, they have solid core. Research at north western university are using gold particles to develop ultra-sensitive detection systems for DNA and protein markers associated with many forms of cancer including breast, prostate cancer.

H) Bucky balls-Bucky ball is common for a molecule called buckminsterfullerene, which is made of 60 carbon atoms formed in shape of hollow ball. Buckyballs and other fullerenes because of their chemistry and their unusual hollow cage-like shape extremely stable and can withstand high temperatures. Buckyballs may see widespread use in future products and applications, from drug delivery vehicles for cancer therapy to ultra-hard coating and military harmer. Bucky ball-Antibody combination delivers antitumor drugs.

I) Carbon nanotubes-Carbon nanotubes show no significant toxicity when they have modified so as to be soluble in aqueous, body type fluids. Carbon nanotubes can be modified to circulate well within the body. Such modifications can be accomplished with covalent or non-covalent bonding. Modifications can increase or decrease circulation time within the body. They enter readily into the cells. Large molecules which circulate slowly can leak into and accumulate in cancer cells. Carbon nanotubes carrying active agents have been demonstrated in animal studies to do this. Researchers have also used carbon tubes to deliver the precursors of active drug, which they call a prodrug, eg: Cisplatin.

\section{Niosomes [12]}

In niosomes, the vesicles forming amphiphile is a non-ionic surfactant such as Span-60 which is usually stabilized by addition of cholesterol and small amount of anionic surfactant such as dicetyl phosphate. Niosomes are preferred over liposomes because the former exhibit high chemical stability and economy. Surfactant forming niosomes are biodegradable, non-immunogenic and biocompatible. Incorporating them into niosomes enhances the efficacy of the drug, such as nimesulide, flurbiprofen, piroxicam, ketoconazole and bleomycin exhibit more bioavailability than the free drug.

\section{Transdermal drug delivery system [12-13]}

Transdermal drug delivery is self-contained, discrete dosage forms which, applied to the intact skin, and deliver the drug at controlled rate to the systemic circulation the merits of delivering drugs across the skin to achieve systemic effects are as it avoids first-pass metabolism, gastrointestinal incompatibility. Helps in improving physiological and pharmacological response. Termination of therapy is easy at any point of time. Greater patient compliance due to less dosing profile. It provides suitability for self-administration and helps in enhancing therapeutic efficacy.

\section{Mucoadhesive drug delivery systems [14]}

In pharmaceutical sciences, when the adhesive attachment is to mucus or a mucous membrane, the phenomenon is referred to as mucoadhesion. The potential of mucoadhesive polymers shown in ocular, nasal, vagina and buccal drug delivery system which leads to significantly prolonged residence time of sustained-release delivery systems on this mucosal membranes.

\section{Sonophoresis [7-9]}

Sonophoresis is a localized, nonvasive, convenient , and rapid method of delivering low molecular weight drugs as well as macromolecules into the skin. It is a process which exponentially increases the absorption of topical compounds into the epidermis, dermis and skin appendages by ultrasonic energy. Sonophoresis is considered to enhance drug delivery through a combination of thermal, chemical and mechanical alterations within the skin tissue. Ultrasound parameters such as treatment duration, intensity, and frequency are all known to affect percutaneous absorption, with the latter being the most important. Ultrasound at various frequencies in the range of $20 \mathrm{kHz}-16 \mathrm{MHz}$ with intensities of up to $3 \mathrm{~W} / \mathrm{cm} 2 \mathrm{has}$ been used for Sonophoresis. Sonophoresis occurs because ultrasound waves stimulate micrevibrations within the skin epidermis and increase the overall kinetic energy of molecules making up topical agents. The ultrasound probably enhances drug transport by cavitation, micro-streaming, and heating. Sonophoresis is widely used in hospitals to deliver drugs through the skin. Application of ultrasound to the skin increases its permeability (Sonophoresis) and enables the delivery of various substances into and through the skin. Sonophoresis is also used in physical therapy. Reverse ultrasound technology may also be used for the extraction of interstitial fluid samples for analysis. So, In addition to its effects in delivering compounds into the skin, Sonophoresis is being investigated as a way of drawing compounds such as glucose out of the skin.

\section{Iontophoresis (IP) [20]}

Iontophoresis is an electrochemical method that enhances the transportation of some solute molecule by creating a potential gradient through the skin with an applied electrical current or voltage. It induces increased migration of ionic drugs into skin by electrostatic repulsion at active electrode. Negative ions are delivered by cathode and positive ion by anode. Typical iontophoresis devices consist of battery, microprocessor controller, drug reservoir and electrodes.

\section{Phonophoresis [19-21]}

Phonophoresis is the transportation of drugs through the skin using ultrasound. It is the combination of ultrasound therapy with topical drug therapy to achieve therapeutic drug concentrations at selected sites in the skin. It is widely used by physiotherapists. Today that product is applied to the skin and some time is allowed for drug to begin absorption into the skin. Then the ultrasound unit is applied.

\section{Miniaturized drug delivery system for intracorporeal use $[15,16]$}

Modern diagnostic and therapeutic procedures address the quality of patient care and aimed at reducing pain and discomfort. Research in microendoscopy is devoted to miniaturization of devices and to 
integration of microsystems into tip of endoscope in order to increase its functionalities. Endoscopic wireless devices usually refer to as endoscopic pills.

\section{Erythrocytes [17]}

Erythrocytes are biocompatible, biodegradable, posse's long circulation half-life and can be loaded with variety of biologically active substances. Erythrocytes loaded drug is one of the potential systems for delivery of drugs and enzymes. Carrier erythrocytes are prepared by collecting blood sample from the organism of interest and separating erythrocytes from the plasma. By using various physical and chemical methods cells are broken and drug is entrapped into erythrocytes, finally they are resealed and resultant carriers are then called as "resealed erythrocytes". Upon reinjection, the drug-loaded erythrocytes serve as slow circulation depots, targets the drug to reticuloendothelial system.

\section{Chronotherapeutics [18]}

Chronotherapeutics is a method of treatment in which in-vivo drug availability is timed to match rhythms of disease, in order to optimize therapeutic outcomes and minimizes side effects. Controlled release formulation can be divided into subgroups such as rate controlled release, delayed-release and pulse release formulations. Enteric coatings have traditionally been used as layer device in the treatment of Parkinsonism patients using l-dopa/benzarazide.

\section{Supramolecular drug delivery systems [20]}

A supramolecular system is composed of two or more molecular entities held together and organized by means of intermolecular non-covalent binding interactions. Supramolecular structures involving macrocyclic compounds have attracted models for understanding natural supramolecular self-assembly molecular recognition and precursors for designing novel nonmaterial for electronics, biomedical and pharmaceutical applications.

\section{Microencapsulation [16]}

Microencapsulation is the process involves small droplets or particles of liquid or solid material are surrounded or coated by a continuous film of polymeric materials. The controlled drug delivery system has used to solve the problems associated with conventional therapy and to improve the therapeutic efficacy of a given drug. The maximum therapeutic efficacy can be achieved by delivering of the active agent in the optimal rate to the target tissue. Microencapsulation process convert the liquids to solids, changing the colloidal and surface properties, providing environmental protection and controlling the release characteristics of different coated materials. Some of these properties can be achieved by micro packaging techniques but in microencapsulation the small coated particles are used to make a wide variety of dosage forms and have not been feasible. Novel drug delivery systems which were initiated with the course of optimizing the bioavailability by the modification of the bioavailability of the drug concentration in blood. With the sustained and controlled release products, drug therapy can be improved that is the common goal achieved over with their non sustained and controlled release with the same drug. Microencapsulated products (microparticles) are the small entities that have an active agent know as the core material surrounded by a shell known as the coating material or embedded into a matrix structure. Most micro particle shells are of organic polymers, but waxes and lipids are also used. Generally the size of the microencapsulated products (microparticles) is considered as larger than 1 micrometer and up to 1000 micrometers in diameter. Commercially available microparticles contained $10-90 \% \mathrm{w} / \mathrm{w}$ core. A number of core materials can be encapsulated like those live cells, adhesives, flavors, agrochemicals, enzymes, pharmaceuticals. The more recent result of pharmaceutical research is that the absorption rate of a drug can be controlled by controlling its rate of release from the dosage form. The controlled released dosage forms are so designed and formulated as having the sustained action, sustained release, prolonged action, delayed action and timed release medication. This has been done by developing the new drug entities, discovering of new polymeric materials that are suitable for prolonging the drug release, safety, improvement in therapeutic efficacy.

\section{Osmotically controlled drug delivery systems [14-19]}

Development of osmotic drug delivery systems was pioneered by Alza and it holds major number of the patents analyzed and also markets several products based on osmotic principle. Osmotic pressure is used as driving force for these systems to release the drug in a controlled manner. Osmotic drug delivery technique is the most interesting and widely acceptable among all other technologies used for the same. These systems can be used for both routes of administration i.e. oral and parenteral. Oral osmotic systems are known as gastro-intestinal therapeutic systems (GITS). Parenteral osmotic drug delivery includes implantable pumps.

\section{Classification of osmotic drug delivery system}

Many forms of osmotic pumps are reported in the literature but, in general they can be divided in oral and implantable systems. Osmotic Drug Delivery Devices fall in two categories:

\section{Implantable \\ The Rose and Nelson Pump \\ Higuchi Leeper Pump \\ Higuchi Theuwes pump \\ Implantable Miniosmotic pump}

\section{Oral osmotic pump}

\section{Single chamber osmotic pump: Elementary osmotic pump}

Multi-chamber osmotic pump: Push-pull osmotic pump, Osmotic pump with non expanding second chamber

Specific types: Controlled porosity osmotic pump, Osmotic bursting osmotic pump, Liquid OROS, Delayed Delivery Osmotic device, Telescopic capsule, Oros ct (colon targeting), sandwiched oral therapeutic system, Osmotic pump for insoluble drugs, Monolithic osmotic system and OSMAT

\section{Advantages [14]}

Osmotic drug delivery system for oral and parenteral use offer a distinct and practical advantage over other means of delivery.

- They typically give a zero-order release profile after an initial lag.

- Deliveries may be delayed or pulsed if desired.

- Drug release is independent of gastric $\mathrm{pH}$ and hydrodynamic condition.

- They are well characterized and understood.

- The release mechanisms are not dependent on the drug.

- A high degree of in vitro and in vivo correlation (IVIVC) is obtained in osmotic systems.

- The rationale for this approach is that the presence of water in GIT is relatively constant, at least in terms of the amount required for activation and controlling osmotically base technologies.

- Higher release rates are possible with osmotic systems compared with conventional diffusion-controlled drug delivery systems.

- The release from osmotic systems is minimally affected by the presence of food in the gastrointestinal tract.

- The release rate of osmotic systems is highly predictable and can be programmed by modulating the release control parameters.

\section{Disadvantages $[14,15]$}

- Expensive

- If the coating process is not well controlled there is a risk of film defects, which results in dose dumping

- Size hole is critical 
- $\quad$ Dose dumping

- Retrival therapy is not possible in the case of unexpected adverse events.

\section{Fast dissolving tablet [22]}

A novel concept of fast dissolving tablet offers ease of ora administration which enhances patient compliance and also reduces the dosage frequency. This tablet format is designed to allow administration of oral solid dosage form in absence of water or fluid intake. Such tablets readily dissolve or disintegrate in saliva generally within less than 60 seconds. When putting on tongue, this tablet disintegrates, and release the drug instantaneously.

\section{Novel drug delivery system: in herbal formulations [17]}

In the past few decades, considerable attention has been focused on the development of novel drug delivery system (NDDS) for herbal drugs. The novel carriers should ideally fulfill two aims. Firstly, it should deliver the drug at a rate directed by the needs of the body, over the period of treatment. Secondly, it should channel the active entity of herbal drug to the site of action. The variety of novel herbal formulations like polymeric nanoparticles, nanocapsules, liposomes, phytosomes, nanoemulsions, microsphere, transferosomes, and ethosomes has been reported using bioactive and plant extracts.

\section{CONCLUSION}

Novel drug delivery system represents a promising drug delivery system of controlled and targeted release. The emergence of conventional to novel drug delivery system is likely to have a significant impact on drug delivery sector, affecting just about every route of administration from oral to injectable. And the payoff for doctors and patients should be lower drug toxicity, reduced cost of treatments, improved bioavailability, and reduces dosage frequency and an extension of the economic life of proprietary drugs. This would allow earlier and more personalized diagnosis and therapy, improving the effectiveness of drug treatments and reducing side effects. In addition, Novel drug delivery system is a promising platform technology for the synthesis of molecular-specific contrast agents. In NDDS nanoparticles and other formulations as liposomes, neosomes etc have great potentials, being able to convert poorly soluble, poorly absorbed and labile biological active substance into promising deliverable drugs. Now a step has forwarded towards to implement novel drug delivery system in herbal formulations.

Global market for advanced drug delivery systems amounted to $\$ 134.3$ billion in 2008, and was projected to increase to $\$ 139$ billion in 2009. The estimate for 2014 is $\$ 196.4$ billion, for a compound annual growth rate (CAGR) of $7.2 \%$ in the 5 -year period. The largest segment of the market is targeted drug delivery, which reached $\$ 50.9$ billion in 2009 and is expected to increase to $\$ 80.2$ billion in 2014, for a CAGR of 9.5\%. Sustained-release products have the second-largest market share, with estimated sales of $\$ 36.1$ billion in 2009 and $\$ 45.8$ billion in 2014, for a CAGR of $4.9 \%$. Benefits for short half-life drugs, sustained release can mean less frequent dosing and thus better compliance reduce variations in plasma/blood levels for more consistent results.

\section{AUTHORS CONTRIBUTIONS}

All the author have contributed equally

\section{CONFLICT OF INTERESTS}

Declare none

\section{REFERENCES}

1. Torchilin VP. Structure and design of polymeric surfactantbased drug delivery systems. J Controlled Release 2001;73:137-72.

2. Muller Goymann CC. Physicochemical characterization of colloidal drug delivery systems such as reverse micelles, vesicles, liquid crystals, and nanoparticles for topical administration. Eur J Pharm Biopharm 2004;58:343-56.

3. Haag R. Supramolecular drug-delivery systems based on polymeric core-shell architectures. Angew Chem Int Ed 2004;43:278-82.

4. S Varun, R Kapoor, K Preet. Taste masking and formulation of ofloxacin rapid disintegrating tablets and oral suspension. Indian J Pharm Educ Res 2009;43:150-5.

5. Dwarakanadha Reddy P, Swarnalatha D. Recent advances in novel drug delivery systems. Int J Pharm Tech Res 2010;2:2025-7.

6. Verma RK, Krishna DM, Garg S. Formulation aspects in the development of osmotically controlled oral drug delivery systems. J Controlled Release 2002;79:7-27.

7. Zentner GM, McClelland GA, Sutton SC. Controlled porosity solubility-and resin-modulated osmotic drug delivery systems for the release of diltiazem hydrochloride. J Controlled Release 1991;16:237-44.

8. Lindstedt B, Ragnarsson G, Hjartstam J. Osmotic pumping as a release mechanism for membrane-coated drug formulations. Int J Pharm 1989;56:261-8.

9. Ghosh T, Ghosh A. Drug delivery through the osmotic systemsan overview. J Appl Pharm Sci 2011;2:38-49.

10. Reddy PD, Swarnalatha D. Recent advances in novel drug delivery systems. IJPTR 2010;2:2025-7.

11. Muller CC. Physicochemical characterization of colloidal drug delivery systems such as reverse micelles, vesicles, liquid crystals and nanoparticles for topical administration. Eur J Pharm Biopharm 2004;58:343-56.

12. Arunachalam A, karthikeyan M, Vinay Kumar D. Transdermal drug delivery system: a review. Curr Pharma Res 2010;1:70-81.

13. Sharma B, Saroha K, Yadav B. Sonophoresis: an advanced tool in the transdermal drug delivery system. IJCPR 2011;3:89-97.

14. Gupta S, Singh RP, Sharma R, Kalyanwat R, Lokwani P. Osmotic pumps: a review. IJCP 2011;6:1-8.

15. Patel H, Patel U, Kadikar H, Bhimani B, Daslaniya D, Patel G. A review on osmotic drug delivery system. IRJP 2012;3:88-94.

16. Kumar A, Sharma P, Banik A. Microencapsulation as novel drug delivery system. Int Pharm Sci 2011;1:1-7.

17. Saraf AS. Applications of novel drug delivery system for herbal formulations. Fitoterapia 2010;81:680-9.

18. He ZF, Liu DY, Zeng S, Ye JT. Study on preparation of ampelopsin liposomes. China J Chin Mater Med 2008;33:27-30.

19. Rane S, Prabhakar B. Influence of liposome composition on paclitaxel entrapment and $\mathrm{pH}$ sensitivity of liposomes. IJPTR 2009;1:914-7.

20. Godin B, Touitou E. Mechanism of bacitracin permeation enhancement through the skin and cellular membrane from an ethosomal carrier. J Controlled Release 2004;94:365-79.

21. Jzu-Hui Wu, Feng-Lin Yen, Liang-Tzung Lin, Tong-Rong Tsai, ChunChing Lin. Preparation, physicochemical characterization, and antioxidant effects of quercetin nanoparticle. IJP 2008;346:160-8.

22. Sampath Kumar KP, Bhowmik D, Chiranjib Chandira M, Tripathi KK. Innovations in sustained release drug delivery system and its market opportunities. J Chem Pharm Res 2010;2:349-60. 